\title{
Assimilation-contrast effects in impression formation 1
}

\author{
MARTIN F. KAPLAN, Northern Illinois \\ University, DeKalb, Ill. 60115
}

\begin{abstract}
Anchoring effects in impression formation were studied as a function of distance between anchor and test stimulus, and differentiation of judge. Results uniformly lacked support for a perceptual assimilation-contrast approach to context effects in that contrast was not associated with either greater distance between anchor and test or maximum differentiation of judge. Consistent assimilation, or positive context effects, were supportive of an averaging model analysis of context effects in impression formation.
\end{abstract}

Research in context effects, or anchoring in social perception, has suggested two alternative descriptions of findings. The first, following from anchoring effects found in psychophysics, predicts assimilation, or displacement of rating of test stimulus toward the anchor, when test and anchor (or context) are relatively close judgmentally, and contrast, or displacement away from the anchor, when test and anchor are relatively distant from one another (Sherif \& Hovland, 1961). The prime variable in determining distance between test and context, and therefore between assimilation and contrast effects, is number of categories of description intervening between the two. Presumably, the process of assimilation or contrast is a perceptual one, i.e., stimuli are perceived as being more positive or negative due to anchoring.

The second approach suggests that the rating given to a test stimulus in context is an averaged function of its context-free rating and the overall person impression (Anderson \& Lampel, 1965). Thus, it is the rating assigned to a trait that is affected by extreme anchors or contexts, and not its perceptual meaning. Further, the averaging formulation predicts only positive context effects, or assimilation, the extent of which is a function of discrepancy between overall impression and test trait.

Anchors may derive from one or both of two sources: contextual stimuli or initial position of the judge. Findings on the effects of contextual stimuli have been varied; Berkowitz (1960), for example, found contrast effects when judging neutral statements that were preceded by uniformly extreme statements, while Anderson \& Lampel (1965) found assimilation effects to vary linearly with test-context discrepancy. The latter authors account for Berkowitz's
Table 1

Mean Test Trait Ratings as a Function of Context Traits and Judge Differentiation

\begin{tabular}{|c|c|c|c|c|c|}
\hline \multirow{2}{*}{$\begin{array}{c}\text { Test } \\
\text { Trait }\end{array}$} & \multirow[b]{2}{*}{ Judge } & \multicolumn{4}{|c|}{ Context } \\
\hline & & L & $\mathbf{M}-$ & M+ & $\mathbf{H}$ \\
\hline $\mathbf{M}-$ & $\begin{array}{l}L D \\
H D\end{array}$ & $\begin{array}{l}6.2 \\
6.7\end{array}$ & $\begin{array}{l}6.7 \\
6.8\end{array}$ & $\begin{array}{l}6.8 \\
6.8\end{array}$ & $\begin{array}{l}9.1 \\
8.1\end{array}$ \\
\hline $\mathrm{M}+$ & $\begin{array}{l}\text { LD } \\
\text { HD }\end{array}$ & $\begin{array}{r}11.5 \\
9.9 \\
\end{array}$ & $\begin{array}{l}12.3 \\
13.3\end{array}$ & $\begin{array}{l}13.6 \\
13.9 \\
\end{array}$ & $\begin{array}{l}14.2 \\
13.9\end{array}$ \\
\hline
\end{tabular}

contrast findings in terms of rating artifacts associated with sequential presentation. With respect to initial position of the judge (here the judge is considered as an anchor), and his distance from the test stimulus, findings have ranged from both assimilation and contrast contingent on distance (Hovland et al, 1957) to assimilation alone (Blanchard, 1966). In addition to varying the judge's initial position in relation to the test stimulus, distance may also vary with the ability to discriminate among stimuli. Berkowitz (1960) suggests that the more discriminating the judge, i.e., the greater the number of categories of description he is likely to use, the greater the psychological distance between test and context, and hence, contrast effects are more likely to occur.

The present study represents a further test of the implication that increased discrimination is associated with increased probability of contrast. While studies have manipulated discrimination by instructions to presume similarity or dissimilarity with the stimulus person (Blanchard, 1966), or to relate test and context to either the same or different persons (Berkowitz, 1960), the difficult assumption must be made that either presuming similarity or forming a unitary impression reduces distance between anchor and test. Findings were also equivocal, assimilation effects being found in the former study, contrast effects in the latter. In a more direct attempt at manipulating judge discrimination, Atkins et al (1965) found no differential tendencies for anchoring effects between high and low discriminators, but unfortunately, discrimination was not defined independent of the social-judgment task.

In the present experiment, judges were selected on the basis of their characteristic range of responses in describing others. Following Sherif \& Hovland (1961), it would be expected that ratings of moderately favorable stimuli would be displaced toward the context when intervening distance was minimal, and away from the context when distance was maximal. Further, it would be expected that contrast effects would be most pronounced in highly discriminating judges, and assimilation effects maximized in low discriminators. On the other hand, a linear averaging model (Anderson \& Lampel, 1965) predicts assimilation regardless of test-context distance or judge's discrimination ability,

\section{SUBJECTS}

Approximately 100 female volunteers from an introductory psychology course were asked to list all the words they could think of in a $7 \cdot \mathrm{min}$ span which could be used to describe others. It was assumed that the number of descriptive words available for describing others would reflect the number of categories available for social judgment, and consequently, for discrimination. The extent to which one may make distinctions among social objects thus is assumed to be limited by the number of responses typically available to him (cf. Chapanis, 1965), as reflected in his productivity of behavioral labels. In an earlier experiment (Kaplan, 1968), number of words elicited acted as an index of ability to differentiate between social stimuli, providing support for this thesis. After excluding repetitions, females having more than the median number of words were designated as high differentiators (HD), those with less were designated as low differentiators (LD). Fifteen Ss from each group were randomly chosen to participate in the study.

\section{PROCEDURE}

Context traits of high $(\mathrm{H})$, moderately high (M+), moderately low (M-), or low (L) favorability were randomly chosen from a list of prescaled traits (Anderson, 1968). These were randomly paired with $M^{+}$or $M_{-}$ test traits so that eight sets of two context and one test stimuli each were constructed. Three replications of the 2 by 4 test-context matrix were constructed, each trait being selected without replacement. In addition to the 24 experimental sets, 6 practice and 12 filler sets were constructed, the latter consisting of uniformly $\mathrm{H}, \mathrm{M}+, \mathrm{M}-$, and $\mathrm{L}$ stimuli. Traits were randomized within sets and typed on slides for simultaneous presentation, and the order of presentation of sets was also randomized. $S$ was told that each set represented traits of a given person, each trait ascribed by a different acquaintance. Upon presentation of a given set, $S$ was asked to rate the stimulus person's likability on a 7-point scale. After a pause, $S$ was asked to rate the likability of the test trait in this individual, this time on a 20-point scale.

\section{RESULTS AND DISCUSSION}

Mean ratings of $M-$ and $M+$ test traits in each of the four contexts for the two groups of Ss are found in Table 1. M+ test traits 
were generally rated more favorably than M- test traits, and mild positive context effects may be observed for both judge groups and for both test traits. Differentiation of the judges does not appear to be a significant factor. These observations were supported by an analysis of variance in which the only significant effects were due to test traits $(F=219.5$, $\mathrm{df}=1 / 196, \mathrm{p}<.01)$ and context $(\mathrm{F}=8.8$, $\mathrm{df}=3 / 196, p<.01)$. The Judge Differentiation by Context interaction predicted by Berkowitz's discussion of differentiation and anchoring effects was lacking $(F=1.2, \mathrm{df}=3 / 196)$.

Results are consistent with an averaging-model analysis of context effects in that positive context effects were found, and these were essentially similar for both test stimuli, i.e., the Test by Context interaction was insignificant $(F=2.5$, df $=3 / 196)$. The perceptual assimilation-contrast model, on the other hand, received lack of support on two counts; first, judges who have demonstrated greater differentiation in social perception failed to show the predicted contrast effects, and second, test-trait/context pairings with the greatest distance (i.e., $\mathbf{M}-, \mathrm{H} ; \mathrm{M}+, \mathrm{L}$ ) did not result in contrast. The latter negative finding is consistent with the positive context effects reported by Anderson (1966) in test-context pairings of even greater disparity $(\mathrm{H}, \mathrm{L}$ and $\mathrm{L}, \mathrm{H})$, and raises doubts as to the existence of both assimilation and contrast effects in judgment of social stimuli.

The possibility exists that the presence of perceptual contrast is contingent on whether the anchor consists of contextual stimuli or initial attitude; Atkins \& Bieri (1968), for example, found consistent assimilation when contextual stimuli were considered, but, in intermittant responding, found an initial contrast with judges' initial attitude. It is significant, however, that this contrast dissipated with succeeding trials. Atkins (1966), in a design similar to that in which Berkowitz (1960) reported contrast effects, sequentially presented extreme statements prior to neutral ones, alternative pro and con anchors on each trial. As in the Atkins and Bieri study, initial contrast effects were replaced with the assimilation predicted by an averaging process as trials progressed. Only when anchors are univalent over trials in sequential presentation will contrast be noted, this contrast being due more to the rating scale imposed on $S$ than to perceptual changes. In the Bieri \& Atkins (1968) and Atkins (1966) studies, alternation of anchors over trials served to provide $S$ with a wider sample of rating categories than the unipolar presentation used by Berkowitz (1960). When S has the full-scale range in which to assign ratings, the artifact of contrasted ratings apparently is eliminated. In simultaneous presentation, width of rating samples is provided by practice and filler sets.

The presence of assimilation and contrast effects thus appears to be contingent on procedural conditions. The former requires the formation of a composite judgment (cf. Anderson \& Lampel, 1965), while the latter requires sequential presentation of stimuli, univalent anchors, and restricted response scales. Upshaw (1969) has suggested, on the basis of obtained category width and response dispersion in anchoring studies, that context effects reflect scaling phenomena rather than real changes in perception. He proposes that the contrast effects found by Sherif and his associates are due to the limited categorical response language provided by a narrow range of anchors and do not represent a shift in the affective value of test stimuli. Similarly, Anderson (1966) suggests that context effects reflect a shift in the rating of stimuli and not phenomenological meaning. Thus, both contrast and assimilation effects have been considered artifacts in the sense that neither represents a shift in perception but rather a shift in reference scale, the direction of which depends on the range of scale values effectively provided by the anchor.

While contrast effects due to contex tual anchors, when observed, may be at tributed to artifacts associated with stimulus presentation, the question remains whether such judge characteristics as differentiation can affect anchoring tendencies. The present findings, as well as the fact that consistent anchoring tendencies in Ss cannot be found across stimulus dimensions (Atkins, Meyers, \& Kujala, 1965), argue against this. Instead, it is proposed that perceptual contrast and assimilation, with its implication of differential effects of test-context distance, is inapplicable as an explanatory device in the study of context effects in impression formation.

\section{REFERENCES}

ANDERSON, N. H. Component ratings in impression formation. Psychonomic Science, $1966,6,279-280$.

ANDERSON, N. H. Likableness ratings of 555 personality-trait words. Journal of Personality \& Social Psychology, 1968, 9, 272-279.

ANDERSON, N. H., \& LAMPEL, A. Effect of context on ratings of personality traits. Psychonomic Science, 1965, 3, 433-434.

ATKINS, A. L. Own attitude and discriminability in relation to anchoring effects in judgment. Joumal of Personality \& Social Psychology, 1966, 4, 497-507.

ATKINS, A. L., \& BIERI, J. Effects of involvement level and contextual stimuli on social judgment. Joumal of Personality \& Social Psychology, 1968, 9, 197-204.

ATKINS, A. L., MEYERS, B. A., \& KUJALA, K. Anchoring tendencies as cognitive styles. Paper presented at meeting of the Eastern Psychological Association, Atlantic City, 1965. BERKOWITZ, L. The judgmental process in personality functioning. Psychological Review, $1960,67,130-142$.

BLANCHARD, W. A. Assimilation and contrast in interpersonal prediction with control for the interaction of real similarity and differential accuracy. Journal of Personality \& Social Psychology, 1966, 3, 567-573.

CHAPANIS, $A$. Color names for color space. American Scientist, 1965, 53, 327-346.

HOVLAND, C. I., HARVEY, O., \& SHERIF, M. Assimilation and contrast effects in reaction to communications and attitude change Joumal of Abnormal Social Psychology, 1957, 55, 244-252.

KAPLAN, M. F. Differentiation among targets in social perception as a function of response hierarchy. Psychonomic Science, 1968, 10, 227-228.

SHERIF, M., \& HOVLAND, C. J. Social judgment. New Haven: Yale University Press, 1961.

UPSHAW, H. S. The personal reference scale: An approach to social judgment. In L. Berkowitz (Ed.), Advances in experimental social psychology. Vol. 4. New York: Academic Press, 1969. Pp. 315-371.

NOTE

1. This research was largely supported by a faculty research grant awarded by the College Council, College of Liberal Arts \& Sciences, Northern Illinois University.

\section{Paced vs. unpaced paired-associate recall ${ }^{1}$}

JOHN P. HOUSTON, University
California, Los Angeles, Calif. 90024

In an attempt to eliminate a potentially confounding factor in the comparison of of paced and unpaced recall procedures, Ss first learned either an $A-B$ or successive $A-B, A-C$ paired-associate lists. One week later, Ss were asked to recall the first-list responses. Recall was either paced or unpaced. In 